\title{
On Kalman Filtering over Fading Wireless Channels with Controlled Transmission Powers *
}

\author{
$\underline{\text { Daniel E. Quevedo }}^{\mathrm{a}}$, Anders Ahlén ${ }^{\mathrm{b}}$, Alex S. Leong ${ }^{\mathrm{c}}$, Subhrakanti Dey $^{\mathrm{c}}$ \\ ${ }^{\text {a }}$ School of Electrical Engineering \& Computer Science, The University of Newcastle, NSW 2308, Australia \\ ${ }^{\mathrm{b}}$ Department of Engineering Sciences, Signals and Systems, Uppsala University, P. O. Box 534, SE-751 21 Uppsala, Sweden \\ ${ }^{\mathrm{c}}$ Department of Electrical and Electronic Engineering, The University of Melbourne, Parkville, VIC 3052, Australia
}

\begin{abstract}
We study stochastic stability of centralized Kalman filtering for linear time-varying systems equipped with wireless sensors. Transmission is over fading channels where variable channel gains are counteracted by power control to alleviate the effects of packet drops. We establish sufficient conditions for the expected value of the Kalman filter covariance matrix to be exponentially bounded in norm. The conditions obtained are then used to formulate stabilizing power control policies which minimize the total sensor power budget. In deriving the optimal power control laws, both statistical channel information and full channel information are considered. The effect of system instability on the power budget is also investigated for both these cases.
\end{abstract}

Key words: State estimation, fading channels, packet dropouts, stochastic stability, power control.

\section{Introduction}

The interest in estimation and control over wireless communication channels has increased tremendously. The rapid evolution of wireless sensor networks, see e.g., [10,14, 18, 39] has made wireless sensors (WSs) cheap and reliable enough to be brought into commercial use. WSs offer several advantages for industrial control systems, such as, flexibility, low cost, and fast deployment. Furthermore, with WSs electrical contact problems, often regarded as a nuisance in wired systems, are no longer an issue. Furthermore, WSs and actuators can be placed where wires cannot go, or where power sockets are unavailable. The use of wireless communications for state estimation and closed loop control, however, also brings new challenges in system design. In fact, wireless communication channels are frequently subject to time-varying fading and interference, which may lead to packet errors; see, e.g., [15,32,38].

\footnotetext{
‡ This research was supported under Australian Research Council's Discovery Projects funding scheme (project number DP0988601). A preliminary version of parts of this work appears in [34]. Corresponding author D. E. Quevedo. Tel. +61-249217072. Fax +61-2-49601712.

Email addresses: dquevedo@ieee.org (Daniel E. Quevedo), anders.ahlen@signal.uu.se (Anders Ahlén), asleong@unimelb.edu. au (Alex S. Leong), sdey@unimelb.edu.au (Subhrakanti Dey).
}

A key aspect is that fading channels can partially be compensated for by adjusting the transmission power levels; higher transmission power giving less packet errors. Power control has been shown to be a key enabling technology whenever wireless links are used, constituting a versatile means to guarantee quality of service to individual users $[3,6,11,13,16]$. Since in most WS applications, energy is severely limited, power control design involves trading energy consumption for accuracy [4, 29, 30, 44,46].

Kalman filters are widespread in estimation and control applications and the effect of random measurement losses on filter stability has received considerable attention in recent literature. In particular, [41] studied a single-link estimation setup for LTI plants where dropout processes are independent and identically distributed (i.i.d.). Thus, in this modeling paradigm it is assumed that the probability of drops is the same from one transmission to the next. By using a fixed-point argument, [41] established that there exists a critical dropout probability value which separates situations where the expected value of the covariance matrix remains bounded from instances where it diverges, see also $[21,31]$ and [23]. ${ }^{1}$ The latter work examines a state estimation ar-

\footnotetext{
${ }^{1}$ If rather than the expected value of the covariance matrix, other metrics are used, then different critical dropout probabilities will be obtained, see also $[5,17,36]$. Alternatively, the works $[5,9,20,40]$ directly examine the distribution of the covariance matrix.
} 
chitecture with two channels affected by i.i.d. dropouts. The case of Kalman filtering with a single link and where the dropouts are described by a time-homogeneous two-state Markov chain was investigated in [17, 19,40,45], whereas in the recent work [5] also semi-Markov chains are considered. Given the fundamental importance of power control in wireless communications, it is surprising that the role of power control on stability of Kalman filters with random packet dropouts has not yet been investigated. In fact, to the best of the authors' knowledge, the only works which have examined power control design for Kalman filtering with packet dropouts are our own; see, e.g., [28,35]. These works examine the trade-off between energy use and estimation accuracy, but do not investigate stability issues.

In the present work we study state estimation for linear timevarying systems via a Kalman filter performing optimal centralized state estimation at a single gateway. To keep WSs simple, only raw measurements are transmitted to the gateway; c.f., $[12,15,37,40]$. The wireless transmission channels are block-fading and generate random packet loss. The packet loss probabilities depend, in a nonlinear fashion, upon the time-varying channel gains and power levels used by the sensors. The gateway has knowledge on whether received data is error-free or not. Our main purpose is to investigate the role of power control of the transmission power levels to be used by each WS. We establish sufficient conditions which ensure that the Kalman filter covariance matrix is exponentially bounded in norm. The conditions obtained are then used to formulate stabilizing optimal power allocation laws which minimize the total power used by the sensors.

The remainder of this paper is organized as follows: Section 2 introduces the system setup and revises basic aspects on state estimation with packet drops and power control. A sufficient condition for stochastic stability of the estimator is then obtained in Section 3. Section 4 studies decentralized power control laws. Robustness issues are investigated in Section 5. The stability results are used to perform optimum power allocation in Section 6. Numerical studies are included in Section 7. Section 8 draws conclusions. All proofs of technical results are included in appendices.

Notation: We write $\mathbb{R}_{>0}$ for the nonnegative real numbers and $\mathbb{N}_{0}$ for $\{0,1,2, \ldots\}$. For sequences, we use $\{\nu\}_{\mathbb{N}_{0}}$ for $\{\nu(0), \nu(1), \ldots\}$, and $\{\nu\}_{0}^{k}$ for $\{\nu(0), \ldots, \nu(k)\}$. Given a vector $v, \operatorname{diag}(v)$ is the corresponding diagonal matrix; its Euclidean norm is $|v|=\sqrt{v^{T} v}$, where the superscript ${ }^{T}$ refers to transposition. The eigenvalues of a square matrix $M$ are denoted via eigs $(M)$, its trace via $\operatorname{tr} M$, and its spectral norm by $\|M\| \triangleq \sqrt{\max \operatorname{eigs}\left(M^{T} M\right)}$. If $M$ is positive definite (semi definite), then we write $M \succ 0(M \succeq 0)$. The $L \times L$ identity matrix is denoted via $I_{L}$; its $\ell$-th row is denoted via $\delta_{L}^{\ell}$. Given two sets $A$ and $B, A \backslash B \triangleq\{a \in A: a \notin B\}$.

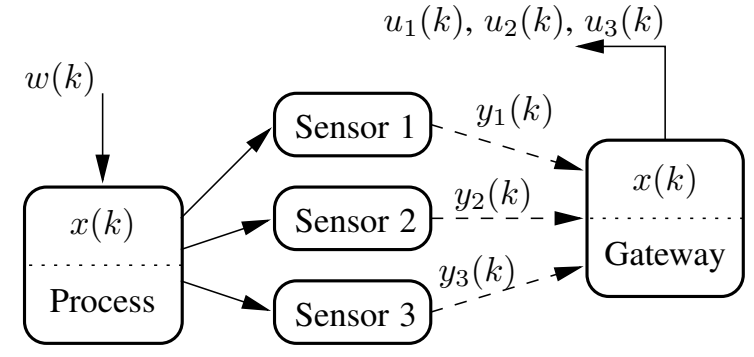

Fig. 1. State estimation with transmission power control of 3 WSs.

The indicator function of a set $A$ is denoted via $\mathbf{1}_{A}$, i.e.,

$$
\mathbf{1}_{A}(a)= \begin{cases}1 & \text { if } a \in A, \\ 0 & \text { if } a \notin A\end{cases}
$$

A function $\theta: \mathbb{R}_{\geq 0} \rightarrow \mathbb{R}_{\geq 0}$ is of class- $\mathcal{K}$, if it is continuous, zero at zero and strictly increasing. We write $\operatorname{Pr}\{\Omega \mid \Delta\}$ for the conditional probability of $\Omega$ given $\Delta$ and $\operatorname{Pr}\{\Omega\}$ for the unconditional probability. The cumulative distribution function (cdf) of a random variable $\nu$ is $F_{\nu}(v) \triangleq \operatorname{Pr}\{\nu \leq v\}$. The expected value of $\nu$ is, thus, given by the LebesgueStieltjes integral $\mathbf{E}\{\nu\}=\int_{\Lambda} z \mathrm{~d} F_{\nu}(z)$, where $\Lambda$ is the support of $\nu ; \mathbf{E}\{\nu \mid \Delta\}$ refers to conditional expectation. A random variable $\nu$, which is Gaussian with mean value $m$ and covariance matrix $\Gamma \succ 0$ is denoted $\nu \sim \mathcal{G}(m, \Gamma)$. At times, we use the same notation for random variables and their realizations. What is meant will depend on the context.

\section{System Setup}

Consider a linear time-varying $n$-dimensional plant model

$$
x(k+1)=A(k) x(k)+w(k) \in \mathbb{R}^{n}, x(0) \sim \mathcal{G}\left(x_{0}, P_{0}\right) .
$$

The driving noise process $\{w\}_{\mathbb{N}_{0}}$ is white, where each $w(k) \sim \mathcal{G}(0, Q(k))$.

To remotely estimate the state sequence $\{x\}_{\mathbb{N}_{0}}, L \geq n$ wireless sensors are used. Each sensor $\ell \in\{1,2, \ldots, L\}$ provides a noisy measurement sequence, say $\left\{y_{\ell}\right\}_{\mathbb{N}_{0}}$, where

$$
y_{\ell}(k)=C_{\ell} x(k)+v_{\ell}(k) \in \mathbb{R}^{m_{\ell}}, \quad m_{\ell} \in \mathbb{N}
$$

and $\left\{v_{\ell}\right\}_{\mathbb{N}_{0}}$ are white with $v_{\ell}(k) \sim \mathcal{G}\left(0, R_{\ell}(k)\right) .^{2}$ Throughout this work, we assume that $\{A\}_{\mathbb{N}_{0}},\{Q\}_{\mathbb{N}_{0}}$ and $\left\{R_{\ell}(k): k \in \mathbb{N}_{0}, \ell \in\{1,2, \ldots, L\}\right\}$ are deterministic and bounded sequences, which are known at the gateway.

The $L$ measurements in (3) are to be transmitted to a single gateway via wireless links. Upon receipt, the signals are used to remotely estimate the state of the system (2), see Fig. 1.

\footnotetext{
2 In addition to measurement noise, $v_{\ell}(k)$ may also describe quantization effects, here modeled as Gaussian and introducing possibly time-varying distortion [27,35].
} 


\subsection{Effects of Channel Fading}

In the present work, we will adopt a block-fading channel gain model, which is a common information theoretic model for fading wireless channels where the channel power gains remain invariant over a block (equivalent to the coherence time of the channel) and may change from block to block [4]. We furthermore assume single measurements $y_{\ell}(k)$ to be a packet, thus, the block length equals the packet transmission time. The associated channel gain processes are denoted via

$$
h_{\ell}(k) \in \Omega_{\ell} \subseteq \mathbb{R}_{\geq 0}, \quad \ell \in\{1,2, \ldots, L\} .
$$

Since the $L$ links are wireless, transmission errors are likely. Faulty packets will be discarded when estimating $\{x\}_{\mathbb{N}_{0}}$; c.f. [26]. To model transmission effects we introduce the $L$ binary stochastic arrival processes $\left\{\gamma_{\ell}\right\}_{\mathbb{N}_{0}}$, where:

$\gamma_{\ell}(k)= \begin{cases}1 & \text { if } y_{\ell}(k) \text { arrives error-free at time } k, \\ 0 & \text { if } y_{\ell}(k) \text { does not arrive error-free at time } k\end{cases}$

The success probabilities of $\left\{\gamma_{\ell}\right\}_{\mathbb{N}_{0}}$ are determined by the propagation environment and transmission power

$$
u_{\ell}(k) \in\left[0, u_{\ell}^{\max }\right]
$$

where $u_{\ell}^{\max }$ is the peak power level. More precisely, we have

$$
\operatorname{Pr}\left\{\gamma_{\ell}(k)=1 \mid h_{\ell}(k)=h, u_{\ell}(k)=u\right\}=f_{\ell}(h u)
$$

where the functions $f_{\ell}(\cdot): \mathbb{R}_{\geq 0} \rightarrow[0,1]$ are monotonically increasing and differentiable, and depend upon the modulation scheme employed; see, e.g., [32,35].

We introduce $\{\gamma\}_{\mathbb{N}_{0}},\{h\}_{\mathbb{N}_{0}}$ and $\{u\}_{\mathbb{N}_{0}}$, where, $\forall k \in \mathbb{N}_{0}$ :

$\gamma(k) \triangleq\left[\begin{array}{c}\gamma_{1}(k) \\ \gamma_{2}(k) \\ \vdots \\ \gamma_{L}(k)\end{array}\right], h(k) \triangleq\left[\begin{array}{c}h_{1}(k) \\ h_{2}(k) \\ \vdots \\ h_{L}(k)\end{array}\right], u(k) \triangleq\left[\begin{array}{c}u_{1}(k) \\ u_{2}(k) \\ \vdots \\ u_{L}(k)\end{array}\right]$.

Example 1 (BPSK with Rayleigh fading) Suppose that binary phase shift keying transmission over i.i.d. block fading additive white Gaussian noise channels is used. Each sensor measurement consists of a packet containing $b$ bits. Since the bit errors are independent within a packet, the success probabilities can be written as, see [32],

$$
f_{\ell}(h u)=\left(\int_{0}^{\sqrt{h u}} \frac{1}{\sqrt{2 \pi}} e^{-t^{2} / 2} \mathrm{~d} t\right)^{b}
$$

and the gains, $h_{\ell}(k)$, are exponentially distributed with $c d f$

$$
F_{h_{\ell}(k)}(h)= \begin{cases}1-e^{-\lambda_{\ell} h}, & \text { if } h \in \mathbb{R}_{\geq 0}, \\ 0, & \text { if } h<0\end{cases}
$$

where $\lambda_{\ell}>0$. For other transmission schemes, see [7].

\subsection{Control of transmission powers}

The main purpose of this work is to study the role of power control for state estimation over wireless channels. It follows from (6) that one can improve transmission reliability and, thus, state estimation accuracy for a given wireless propagation environment, by increasing the transmission powers. However, with WSs, it is of fundamental importance to save energy. Thus, power control design involves a trade-off between transmission error probabilities (and, thus, state estimation accuracy) and energy consumption, see also [35]. We focus on non-linear feedback policies which are implemented at the gateway, see Fig. 1, and are of the form

$$
\begin{aligned}
\kappa_{\ell}: \Omega_{1} \times \cdots \times \Omega_{L} \rightarrow\left[0, u_{\ell}^{\max }\right] \\
\hat{h}(k) \mapsto u_{\ell}(k)=\kappa_{\ell}(\hat{h}(k)),
\end{aligned}
$$

where

$$
\hat{h}(k) \triangleq\left[\hat{h}_{1}(k) \hat{h}_{2}(k) \ldots \hat{h}_{L}(k)\right]^{T} .
$$

are channel gain estimates. ${ }^{3}$ These satisfy

$$
\hat{h}_{\ell}(k)=\left(1+\epsilon_{\ell}(k)\right) h_{\ell}(k), \quad \ell \in\{1, \ldots, L\}
$$

where $\epsilon_{\ell}(k)$ are the relative estimation errors, with support $\Lambda_{\ell} \subset \mathbb{R}$. If we define

$$
\begin{aligned}
\epsilon(k) & \triangleq\left[\epsilon_{1}(k) \epsilon_{2}(k) \ldots \epsilon_{L}(k)\right]^{T} \in \Lambda \\
\Lambda & \triangleq \Lambda_{1} \times \ldots \Lambda_{L}
\end{aligned}
$$

then (9) can be written in compact form as

$$
\hat{h}(k)=\left(I_{L}+\operatorname{diag}(\epsilon(k))\right) h(k)
$$

\subsection{Kalman filtering with Packet Drop-outs}

Throughout this work, we will assume that the packets transmitted from the sensors to the gateway incorporate error detection coding [32]. Hence, the gateway knows whether received packets are correct or not. Thus, at any time $k$, past and present realizations of the process $\{\gamma\}_{\mathbb{N}_{0}}$ are available

\footnotetext{
${ }^{3}$ For example, one could use $\hat{h}(k)=\mathbf{E}\{h(k)\}$, see also, [8].
} 
at the gateway. For state estimation purposes, the system amounts to sampling (2), (3) with stochastic output matrix

$$
C(k)=\mathcal{C}(\gamma(k)) \triangleq\left[\begin{array}{c}
\gamma_{1}(k) C_{1} \\
\gamma_{2}(k) C_{2} \\
\vdots \\
\gamma_{L}(k) C_{L}
\end{array}\right]
$$

and measurements

$$
y(k) \triangleq C(k) x(k)+\left[v_{1}(k)^{T} v_{2}(k)^{T} \ldots v_{L}(k)^{T}\right]^{T} .
$$

The conditional probability distribution of $x(k)$, given $\{\gamma\}_{0}^{k}$ and correctly received packets with sensor measurements up to time $k$, i.e., $\{y\}_{0}^{k}$, is Gaussian. Consequently,

$$
\begin{aligned}
& \hat{x}(k) \triangleq \mathbf{E}\left\{x(k) \mid\{y, \gamma\}_{0}^{k}\right\} \\
& \check{x}(k) \triangleq \mathbf{E}\left\{x(k) \mid\{y, \gamma\}_{0}^{k-1}\right\} \\
& P(k) \triangleq \mathbf{E}\left\{(x(k)-\check{x}(k))(x(k)-\check{x}(k))^{T} \mid\{y, \gamma\}_{0}^{k-1}\right\},
\end{aligned}
$$

satisfy the Kalman filter recursions (see, e.g., [22]):

$$
\begin{aligned}
\hat{x}(k) & =\check{x}(k)+K(k)(y(k)-C(k) \check{x}(k)) \\
\check{x}(k) & =A(k-1) \hat{x}(k-1) \\
P(k+1) & =A(k)\left(I_{n}-K(k) C(k)\right) P(k) A(k)^{T}+Q(k)
\end{aligned}
$$

with initial values $P(0)=P_{0}$ and $\hat{x}(-1)=x_{0}$, and where

$$
\begin{aligned}
K(k) & \triangleq P(k) C(k)^{T}\left(C(k) P(k) C(k)^{T}+R(k)\right)^{-1} \\
R(k) & \triangleq \operatorname{diag}\left(R_{1}(k), R_{2}(k), \ldots, R_{L}(k)\right) \succ 0 .
\end{aligned}
$$

Note that $C(k)$ takes one of $2^{L}$ possible values. The distribution of $C(k)$ depends upon the current channel gains $h_{\ell}(k)$ and the power levels used; see (6) and (13). Thus, $\{C\}_{\mathbb{N}_{0}}$ is a random process, the recursion (14) is stochastic and the error covariance $\{P\}_{\mathbb{N}_{0}}$ becomes a random process.

\section{Stability with Power Controlled Radio-Links}

Due to random packet dropouts, $\{P\}_{\mathbb{N}_{0}}$ in (14) will in general not converge to a unique value as $k \rightarrow \infty$. Depending upon properties of (2) and $\{\gamma\}_{\mathbb{N}_{0}}, \mathbf{E}\{\|P(k)\|\}$ may remain bounded or diverge. This issue of stability of the Kalman filter is of fundamental importance to characterize estimation quality. The special case of Kalman filtering for LTI systems with one or two links and where arrival processes are i.i.d. Bernoulli with fixed success probabilities has been extensively studied; see, e.g., $[23,38]$. A data-fusion strategy with multiple sensors is studied in [43].

We next examine stability of the estimator for more complex cases where the packet dropout processes are time-varying and governed by power controlled fading channels. For that purpose, we make the following assumption:

Assumption 2 The joint process $\{\xi\}_{\mathbb{N}_{0}}$, where

$$
\xi(k)=\left[\begin{array}{c}
h(k) \\
\epsilon(k)
\end{array}\right], \quad k \in \mathbb{N}_{0}
$$

is white, i.e., the random variables $\left\{\xi(k): k \in \mathbb{N}_{0}\right\}$ are independent (but not necessarily identically distributed).

Channel gains are temporally uncorrelated for fading channels for which coherence times (times for which the channel remains invariant) are shorter than the packet transmission times. This assumption will hold in many practical applications, and was considered, e.g., also in [26]. If Assumption 2 holds, then $\left\{\gamma_{\ell}\right\}_{\mathbb{N}_{0}}$ are white. In addition, it follows from (6) and (8) that

$$
\operatorname{Pr}\left\{\gamma_{\ell}(k)=1\right\}=\int_{\Omega \times \Lambda} \varphi_{\ell}(\xi) \mathrm{d} F_{\xi(k)}(\xi),
$$

where $\Omega \triangleq \Omega_{1} \times \cdots \times \Omega_{L}$ and

$$
\begin{aligned}
\varphi_{\ell}(\xi) & =f_{\ell}\left(\delta_{L}^{\ell} h \kappa_{\ell}\left(\left(I_{L}+\operatorname{diag}(\epsilon)\right) h\right)\right) \\
\xi^{T} & =\left[h^{T} \epsilon^{T}\right], \quad h \in \Omega, \epsilon \in \Lambda .
\end{aligned}
$$

Theorem 3, stated below, establishes sufficient conditions for convergence of $\mathbf{E}\{\|P(k)\|\}, k \in \mathbb{N}_{0}$. To state our result, we introduce the random process $\{s\}_{\mathbb{N}_{0}}$, where

$$
s(k)= \begin{cases}1, & \text { if } C(k) \text { has full column-rank } \\ 0, & \text { otherwise }\end{cases}
$$

Theorem 3 Consider state estimation with power controlled radio links as described in Section 2 and suppose that Assumption 2 holds. If there exists $\rho \in[0,1)$, such that:

$$
\operatorname{Pr}\{s(k)=1\} \geq 1-\frac{\rho}{\|A(k)\|^{2}}, \quad \forall k \in \mathbb{N}_{0},
$$

then, $\{P\}_{\mathbb{N}_{0}}$ is exponentially bounded in norm, i.e.,

$$
\mathbf{E}\{\|P(k)\|\} \leq \alpha \rho^{k}+\beta, \quad \forall k \in \mathbb{N}_{0} .
$$

for some $\alpha, \beta \in \mathbb{R}_{\geq 0}$.

Under the hypotheses of the above theorem, (a specific form of) stochastic stability of Kalman filtering when used for state estimation over wireless fading channels with power control of the form (8) will hold.

The process $s(k)$ is a logical function of $\gamma(k)$. In fact, we have

$$
s(k)=\mathbf{1}_{\mathbb{J}}(\gamma(k)),
$$


using (1), and where

$$
\mathbb{J} \triangleq\left\{\Gamma \in\{0,1\}^{L} \subset \mathbb{R}^{L}: \mathcal{C}(\Gamma) \text { has column-rank } n\right\},
$$

see (13). Alas, $\operatorname{Pr}\{s(k)=1\}$ may be hard to quantify since, due to power control, the individual transmission outcomes $\gamma_{\ell}(k), \ell \in\{1,2, \ldots, L\}$ may be correlated. However, as stated in Lemma 5 below, if the transmission outcomes satisfy the following assumption, then $\operatorname{Pr}\{s(k)=1\}$ can be stated in terms of the conditional success probabilities $\varphi_{\ell}$.

Assumption 4 The transmission outcomes $\gamma_{\ell}(k)$ are conditionally independent given $(h(k), u(k))$, i.e.,

$$
\begin{aligned}
\operatorname{Pr}\left\{\gamma_{\ell}(k)=i, \gamma_{p}(k)=j \mid h(k)=h, u(k)=u\right\} \\
=\operatorname{Pr}\left\{\gamma_{\ell}(k)=i \mid h(k)=h, u(k)=u\right\} \\
\times \operatorname{Pr}\left\{\gamma_{p}(k)=j \mid h(k)=h, u(k)=u\right\},
\end{aligned}
$$

for all $k \in \mathbb{N}_{0}, \forall i, j \in\{0,1\}$ and $\forall \ell, p$ in $\{1,2, \ldots, L\}$.

Lemma 5 Suppose that Assumptions 2 and 4 hold, then

$$
\begin{array}{r}
\operatorname{Pr}\{s(k)=1\}=\int_{\Omega \times \Lambda} \sum_{\Gamma \in \mathbb{J}} \prod_{\ell=1}^{L}\left(\left(1-\delta_{L}^{\ell} \Gamma\right)\left(1-\varphi_{\ell}(\xi)\right)\right. \\
\left.+\delta_{L}^{\ell} \Gamma \varphi_{\ell}(\xi)\right) \mathrm{d} F_{\xi(k)}(\xi),
\end{array}
$$

where $\varphi_{\ell}(\xi)$ are defined in (17) and $\mathbb{J}$, in (22).

Remark 6 As already discussed in Section 1, situations where success probabilities are the same from one instant to the next have been extensively studied in the literature. In particular, [20,21,40] have mainly focused on the case where $C_{\ell}$ has full rank. Our result in Theorem 3 complements existing stability results, in that it considers transmission over multiple power controlled wireless channels with timevarying (but white) success probabilities. Naturally, since Theorem 3 is applicable for time-varying systems, it requires strong conditions, namely (19) needs to hold at all instances $k \in \mathbb{N}_{0}$.

Remark 7 It follows from (18) that Theorem 3 disregards the role of measurement updates where $C(k)$ is not full-rank towards reducing the estimation error covariance. Overcoming this limitation remains a topic for future research.

Example 8 Consider a plant model (2) with state dimension $n=2$ and $L=2$ sensors, with $m_{1}=1, m_{2}=2$, and where

$$
C_{1}=\left[\begin{array}{ll}
1 & 0
\end{array}\right], C_{2}=\left[\begin{array}{ll}
1 & 0 \\
1 & 1
\end{array}\right] \Longrightarrow C(k)=\left[\begin{array}{cc}
\gamma_{1}(k) & 0 \\
\gamma_{2}(k) & 0 \\
\gamma_{2}(k) & \gamma_{2}(k)
\end{array}\right]
$$

Thus, $\mathbb{J}=\left\{\left[\begin{array}{ll}0 & 1\end{array}\right]^{T},\left[\begin{array}{ll}1 & 1\end{array}\right]^{T}\right\}$ and $s(k)=\gamma_{2}(k)$. If we suppose that the controller has access to perfect channel esti- mates and that Assumption 2 holds, then $\operatorname{Pr}\{s(k)=1\}=$ $\int_{\Omega \times\{0\}} \varphi_{2}(\xi) \mathrm{d} F_{\xi(k)}(\xi)$, where $\xi=\left[\begin{array}{llll}h_{1} & h_{2} & \epsilon_{1} & \epsilon_{2}\end{array}\right]^{T}$, and $\xi(k)=\left[h_{1}(k) h_{2}(k) \epsilon_{1}(k) \epsilon_{2}(k)\right]^{T}$. Thus, the sufficient condition for stability established in Theorem 3 becomes: $\int_{\Omega} f_{2}\left(\left[\begin{array}{ll}0 & 1\end{array}\right] h \kappa_{2}(h)\right) \mathrm{d} F_{h(k)}(h) \geq 1-\rho /\|A(k)\|^{2}, \forall k \in \mathbb{N}_{0}$, where $h=\left[\begin{array}{ll}h_{1} & h_{2}\end{array}\right]^{T}$ and $h(k)=\left[h_{1}(k) h_{2}(k)\right]^{T}$.

In the sequel, we will take a closer look at how Theorem 3 can be used in various power control architectures.

\section{Decentralized Control Policies}

A special instance of the class of power control policies considered in (8) is that of decentralized control. Here, the power levels of each of the $L$ transmitters are calculated at the gateway, but depend only upon the channel gain estimate of their links to the gateway:

$$
u_{\ell}(k)=\kappa_{\ell}\left(\hat{h}_{\ell}(k)\right), \quad \forall k \in \mathbb{N}_{0}, \ell \in\{1,2, \ldots, L\},
$$

see (10). Such control laws are, in general, easier to implement than centralized ones of the form (8), especially when the number of transmitters is large. In the decentralized case (25), $\varphi_{\ell}(\xi)$ in (17) becomes

$$
\varphi_{\ell}(\xi)=f_{\ell}\left(\delta_{L}^{\ell} h \kappa_{\ell}\left(\left(1+\delta_{L}^{\ell} \epsilon\right) \delta_{L}^{\ell} h\right)\right)
$$

so that, in contrast to (16) we have

$$
\operatorname{Pr}\left\{\gamma_{\ell}(k)=1\right\}=\int_{\Omega_{\ell} \times \Lambda_{\ell}} f_{\ell}\left(h_{\ell} \kappa_{\ell}\left(\left(1+\epsilon_{\ell}\right) h_{\ell}\right)\right) \mathrm{d} F_{\xi_{\ell}(k)}\left(\xi_{\ell}\right),
$$

where

$$
\xi_{\ell}(k)=\left[\begin{array}{c}
h_{\ell}(k) \\
\epsilon_{\ell}(k)
\end{array}\right], \xi_{\ell}=\left[\begin{array}{l}
h_{\ell} \\
\epsilon_{\ell}
\end{array}\right], \quad \ell \in\{1, \ldots, L\} .
$$

As shown in Lemma 9 given below, in the decentralized case, $\operatorname{Pr}\{s(k)=1\}$ becomes a function of the unconditional link probabilities $\operatorname{Pr}\left\{\gamma_{\ell}(k)=1\right\}$ and the result in Lemma 5 can be further simplified. Note that, as in the centralized case (8), with decentralized control, the resulting stability condition (19) involves, in general, more than one channel.

Lemma 9 Suppose that Assumptions 2 and 4 hold, that the control laws are decentralized, see (25), and define

$$
\begin{aligned}
\Phi_{\ell}(k) & \triangleq \operatorname{Pr}\left\{\gamma_{\ell}(k)=1\right\} \\
\Phi(k) & \triangleq\left[\begin{array}{lll}
\Phi_{1}(k) & \Phi_{1}(k) \ldots \Phi_{L}(k)
\end{array}\right] \\
\psi(\Phi(k)) & \triangleq \sum_{\Gamma \in \mathbb{J}} \prod_{\ell=1}^{L}\left(\left(1-\delta_{L}^{\ell} \Gamma\right)\left(1-\Phi_{\ell}(k)\right)+\delta_{L}^{\ell} \Gamma \Phi_{\ell}(k)\right),
\end{aligned}
$$

where $\mathbb{J}$ is as in (22). Then $\operatorname{Pr}\{s(k)=1\}=\psi(\Phi(k))$. 
A particularly simple decentralized control policy consists in using saturated constant gain power controllers for each channel. Here, the aim is to provide a constant success probability by inverting the channel gain, provided sufficient power is available. Such a control policy is described via:

$$
\kappa_{\ell}\left(\hat{h}_{\ell}(k)\right) \triangleq \begin{cases}K_{\ell} / \hat{h}_{\ell}(k) & \text { if } \hat{h}_{\ell}(k) \geq K_{\ell} / u_{\ell}^{\max } \\ u_{\ell}^{\max } & \text { if } \hat{h}_{\ell}(k)<K_{\ell} / u_{\ell}^{\max }\end{cases}
$$

where $K_{1}, K_{2}, \ldots, K_{L} \in \mathbb{R}_{\geq 0}$. Consequently, (27) gives that if Assumption 2 holds, then

$$
\begin{gathered}
\operatorname{Pr}\left\{\gamma_{\ell}(k)=1\right\}=\int_{\Sigma_{\ell}} f_{\ell}\left(K_{\ell} /\left(1+\epsilon_{\ell}\right)\right) \mathrm{d} F_{\xi_{\ell}(k)}\left(\xi_{\ell}\right) \\
+\int_{\left(\Omega_{\ell} \times \Lambda_{\ell}\right) \backslash \Sigma_{\ell}} f_{\ell}\left(h_{\ell} u_{\ell}^{\max }\right) \mathrm{d} F_{\xi_{\ell}(k)}\left(\xi_{\ell}\right),
\end{gathered}
$$

where $\Sigma_{\ell}=\left\{\left[h_{\ell} \epsilon_{\ell}\right]^{T} \in\left(\Omega_{\ell} \times \Lambda_{\ell}\right):\left(1+\epsilon_{\ell}\right) h_{\ell} \geq K_{\ell} / u_{\ell}^{\max }\right\}$. The above expressions can be used for robust control design, i.e., control design which uses knowledge of the $\operatorname{cdf} F_{\epsilon_{\ell}(k)}$.

Example 10 Consider the configuration in Fig. 1 with $L=$ 3 scalar sensors and state dimension $n=2$. Suppose that the matrix $C(k) \in \mathbb{R}^{3 \times 2}$ in (13) has full column-rank if and only if at least two measurements are received. Then, see (21),

$$
\begin{aligned}
& \mathbb{J}=\left\{\left[\begin{array}{lll}
0 & 1 & 1
\end{array}\right]^{T},\left[\begin{array}{lll}
1 & 0 & 1
\end{array}\right]^{T},\left[\begin{array}{lll}
1 & 1 & 0
\end{array}\right]^{T},\left[\begin{array}{lll}
1 & 1 & 1
\end{array}\right]^{T}\right\} \\
& s(k)=\left(1-\gamma_{1}(k)\right) \gamma_{2}(k) \gamma_{3}(k)+\gamma_{1}(k)\left(1-\gamma_{2}(k)\right) \gamma_{3}(k) \\
& +\gamma_{1}(k) \gamma_{2}(k)\left(1-\gamma_{3}(k)\right)+\gamma_{1}(k) \gamma_{2}(k) \gamma_{3}(k) \text {. }
\end{aligned}
$$

Lemma 5 establishes that, if Assumptions 2 and 4 hold,

$$
\begin{aligned}
\operatorname{Pr}\{s(k)= & 1\}=\int_{\Omega \times \Lambda}\left(1-\varphi_{1}(\xi)\right) \varphi_{2}(\xi) \varphi_{3}(\xi) \mathrm{d} F_{\xi(k)}(\xi) \\
& +\int_{\Omega \times \Lambda} \varphi_{1}(\xi)\left(1-\varphi_{2}(\xi)\right) \varphi_{3}(\xi) \mathrm{d} F_{\xi(k)}(\xi) \\
& +\int_{\Omega \times \Lambda} \varphi_{1}(\xi) \varphi_{2}(\xi)\left(1-\varphi_{3}(\xi)\right) \mathrm{d} F_{\xi(k)}(\xi) \\
& +\int_{\Omega \times \Lambda} \varphi_{1}(\xi) \varphi_{2}(\xi) \varphi_{3}(\xi) \mathrm{d} F_{\xi(k)}(\xi) .
\end{aligned}
$$

If the control laws and channel estimators are decentralized, then $\varphi_{\ell}(\xi)$ takes the form (26) and (32) simplifies to

$$
\begin{gathered}
\operatorname{Pr}\{s(k)=1\}=\int_{\Omega_{1} \times \Lambda_{1}}\left(1-\varphi_{1}\left(\xi_{1}\right)\right) \mathrm{d} F_{\xi_{1}(k)}\left(\xi_{1}\right) \\
\times \int_{\Omega_{2} \times \Lambda_{2}} \varphi_{2}\left(\xi_{2}\right) \mathrm{d} F_{\xi_{2}(k)}\left(\xi_{2}\right) \int_{\Omega_{3} \times \Lambda_{3}} \varphi_{3}\left(\xi_{3}\right) \mathrm{d} F_{\xi_{3}(k)}\left(\xi_{3}\right) \\
\quad+\cdots+\cdots+\ldots \\
=\left(1-\Phi_{1}(k)\right) \Phi_{2}(k) \Phi_{3}(k)+\Phi_{1}(k)\left(1-\Phi_{2}(k)\right) \Phi_{3}(k) \\
\quad+\Phi_{1}(k) \Phi_{2}(k)\left(1-\Phi_{3}(k)\right)+\Phi_{1}(k) \Phi_{2}(k) \Phi_{3}(k),
\end{gathered}
$$

in agreement with Lemma 9.

\section{Robustness to Uncertainty in Channel Gains}

We will next turn our attention to nominal designs, i.e., where the control policy is chosen such that stability is guaranteed under the assumption that the channel estimates are error-free. Thus, the control laws $\kappa_{\ell}$ are chosen by only taking into account the nominal quantities:

$$
\begin{aligned}
\hat{\varphi}_{\ell}(h) & \triangleq f_{\ell}\left(\delta_{L}^{\ell} h \kappa_{\ell}(h)\right) \\
\hat{\Phi}_{\ell}(k) & \triangleq \int_{\Omega} \hat{\varphi}_{\ell}(h) \mathrm{d} F_{h(k)}(h) \\
\hat{\Phi}(k) & \triangleq\left[\begin{array}{llll}
\hat{\Phi}_{1}(k) & \hat{\Phi}_{2}(k) \ldots & \hat{\Phi}_{L}(k)
\end{array}\right] .
\end{aligned}
$$

Whilst assuming that channel gains are exactly known is fairly common in the wireless communication community, see also [7,26], it is certainly of interest to investigate robustness of nominal designs to channel estimation errors. In particular, with estimation errors, $\hat{\Phi}(k)$ is only an approximation of the actual success probabilities $\Phi(k)$ as defined in (16) and (29). Robust stability, as per Theorem 3, in the presence of channel estimation errors depends upon the success probabilities of the individual channels. A simple bound is established in Theorem 12 given below, which applies to cases where the functions $f_{\ell}$ and $\kappa_{\ell}$ are continuous.

Assumption 11 There exist class- $\mathcal{K}$ functions $\theta_{\ell}$ and $\sigma_{\ell}$, $\ell \in\{1,2, \ldots, L\}$ such that the following are satisfied:

$$
\begin{aligned}
\left|f_{\ell}\left(\nu_{1}\right)-f_{\ell}\left(\nu_{2}\right)\right| & \leq \theta_{\ell}\left(\left|\nu_{1}-\nu_{2}\right|\right) \\
\left|\kappa_{\ell}\left(v_{1}\right)-\kappa_{\ell}\left(v_{2}\right)\right| & \leq \sigma_{\ell}\left(\left|v_{1}-v_{2}\right|\right)
\end{aligned}
$$

for all $\nu_{1}, \nu_{2} \in \mathbb{R}_{\geq 0}$ and for all $v_{1}, v_{2} \in \Omega_{\ell}$.

Theorem 12 Suppose that Assumption 11 holds, then

$$
\begin{gathered}
\qquad \Phi_{\ell}(k) \geq \hat{\Phi}_{\ell}(k)-\Delta_{\ell}(k), \quad \forall \ell \in\{1,2, \ldots, L\}, \\
\text { where } \Delta_{\ell}(k) \triangleq \int_{\Omega \times \Lambda} \theta_{\ell}\left(\delta_{L}^{\ell} h \sigma_{\ell}(|\operatorname{diag}(\epsilon) h|)\right) \mathrm{d} F_{\xi(k)}(\xi) .
\end{gathered}
$$

If, in addition, $\kappa_{\ell}$ is decentralized (and in (34), one takes $v_{1}, v_{2} \in \Omega_{\ell}$ ), then, see (29),

$$
\operatorname{Pr}\{s(k)=1\} \geq \psi(\hat{\Phi}(k)-\Delta(k))
$$

where $\Delta(k) \triangleq\left[\Delta_{1}(k) \ldots \Delta_{L}(k)\right]$.

The bound established in Theorem 12 can be combined with Theorem 3 to analyze robust stability of nominal control laws. As shown below, if $\kappa_{\ell}$ are decentralized and nonincreasing, then smaller values for $\Delta_{\ell}(k)$, thus giving tighter bounds in (35) and (36), can be found: 
Lemma 13 If Assumption 11 holds and $\kappa_{\ell}$ are decentralized and non-increasing, then (35) and (36) hold with

$$
\Delta_{\ell}(k)=\Delta_{\ell}^{\prime}(k) \triangleq \int_{\Omega_{\ell} \times \Lambda_{\ell}^{+}} \theta_{\ell}\left(h_{\ell} \sigma_{\ell}\left(\epsilon_{\ell} h_{\ell}\right)\right) \mathrm{d} F_{\xi_{\ell}(k)}\left(\xi_{\ell}\right),
$$

where $\Lambda_{\ell}^{+} \triangleq \Lambda_{\ell} \cap \mathbb{R}_{\geq 0}$.

The following corollary to Theorem 12 can be used for robust design, i.e., control design which uses $F_{\xi(k)}$ to obtain stabilizing control in the presence of channel estimation errors. It uses the following definition:

$$
D(k) \triangleq \max _{c \in[0,1]} \nabla \psi(\hat{\Phi}(k)-c \Delta(k)) \Delta(k)^{T},
$$

where $\nabla \psi(\chi)$ is the gradient of $\psi$ at $\chi, \Delta(k)$ is as in Theorem 12 , and $\hat{\Phi}(k)$ is defined in (33).

Corollary 14 Suppose that the hypotheses of Theorems 3 and 12 are satisfied, and that $L$ decentralized control policies have been designed such that, for some $\tilde{\rho} \in[0,1)$,

$$
\psi(\hat{\Phi}(k)) \geq 1-\frac{\tilde{\rho}}{\|A(k)\|^{2}}, \quad \forall k \in \mathbb{N}_{0}
$$

i.e., the nominal system is stable, see Lemma 9 and Thm 3. If

$$
\tilde{\rho}<1-\|A(k)\|^{2} D(k), \quad \forall k \in \mathbb{N}_{0},
$$

then (20) holds for some $\rho \in[0,1)$.

Thus, designs which use $\hat{\Phi}(k)$ giving $\tilde{\rho}<1$ in (38) have inherent robustness with respect to small estimation errors.

Example 15 Suppose that $L=2$ and that $\operatorname{Pr}\{s(k)=1\}=$ $\Phi_{1}(k) \Phi_{2}(k)$, both packets need to be successfully received for $C(k)$ to have full-rank. Direct calculations provide $\nabla \psi(\hat{\Phi}(k)-c \Delta(k))=\left[\hat{\Phi}_{2}(k) \hat{\Phi}_{1}(k)\right]-c\left[\Delta_{2}(k) \Delta_{1}(k)\right]$. Thus, $D(k)=\hat{\Phi}_{2}(k) \Delta_{1}(k)+\hat{\Phi}_{1}(k) \Delta_{2}(k)$ can be used in (39) as a sufficient condition for robust stochastic stability of the Kalman filter with decentralized power control. $\square$

\section{Optimal Power Control Strategies}

In general, state estimation accuracy can be improved by increasing the power on the channels being in a fade. However, such a simple strategy may often waste energy. A better idea is to allocate only as much power to the sensors as is necessary to attain desired performance. A lower bound would then be the allocation of power to the sensors that is sufficient to maintain stability. The result in Theorem 3 motivates one to minimize the nominal average sum power

$$
\bar{U}(k) \triangleq \sum_{\ell=1}^{L} \int_{\Omega} \kappa_{\ell}(h) \mathrm{d} F_{h(k)}(h),
$$

subject to (19). In what follows we investigate three nominally stabilizing power control laws which minimize (40) and are of increasing complexity.

\subsection{Constant Power Levels}

The simplest decentralized power control method consists in using constant power levels $\left\{u_{1}, \ldots, u_{L}\right\}$. By Lemma 9, the optimization problem for the nominal case becomes

$$
\min _{\left(u_{1}, \ldots, u_{L}\right)} \sum_{\ell=1}^{L} u_{\ell}, \quad \text { s.t. } \psi(\hat{\Phi}(k)) \geq 1-\frac{\rho}{\|A(k)\|^{2}},
$$

where $\hat{\Phi}_{\ell}(k)=\int_{\Omega_{\ell}} f_{\ell}\left(h_{\ell} u_{\ell}\right) \mathrm{d} F_{h_{\ell}(k)}\left(h_{\ell}\right)$, see (33).

Example 16 Consider a simple architecture with scalar sensors, where $L=n$, and where the vectors $\left\{C_{1}, \ldots, C_{L}\right\}$ are linearly independent. Thus, $C(k)$ has full rank, if and only if $\gamma_{\ell}(k)=1, \forall \ell \in\{1,2, \ldots, L\}$. In this case $\mathbb{J}=$ $\left\{\left[\begin{array}{lll}1 & 1 & \ldots\end{array}\right]^{T}\right\}$ and (29) gives that $\psi(\hat{\Phi}(k))=\prod_{\ell=1}^{L} \hat{\Phi}_{\ell}(k)$. By taking logarithm of the constraint in (41), we obtain the equivalent problem

$$
\begin{aligned}
\min _{\left\{u_{1}, \ldots, u_{L}\right\}} & \sum_{\ell=1}^{L} u_{\ell} \\
\text { s.t. } & \sum_{\ell=1}^{L} \log \left(\hat{\Phi}_{\ell}(k)\right) \geq \log \left(1-\frac{\rho}{\|A(k)\|^{2}}\right) .
\end{aligned}
$$

The following result shows that under BPSK transmission and Rayleigh fading, in this simple case, the optimal power levels can be found with standard optimization software.

Lemma 17 Consider the communication setup in Example 1. Then (42) is a convex optimization problem.

\subsection{Saturated Constant Gain Power Control}

We will next investigate the saturated power control law of (30), Here, we set $\Omega_{\ell}=\mathbb{R}_{\geq 0}$ for all $\ell \in\{1, \ldots, L\}$. In this case it follows directly from (31) and (29) that

$$
\begin{array}{r}
\hat{\Phi}_{\ell}(k)=\int_{0}^{K_{\ell} / u_{\ell}^{\max }} f_{\ell}\left(h_{\ell} u_{\ell}^{\max }\right) \mathrm{d} F_{h_{\ell}(k)}\left(h_{\ell}\right) \\
+\int_{K_{\ell} / u_{\ell}^{\max }}^{\infty} f_{\ell}\left(K_{\ell}\right) \mathrm{d} F_{h_{\ell}(k)}\left(h_{\ell}\right),
\end{array}
$$

whereas the average sum power becomes

$$
\begin{array}{r}
\bar{U}(k)=\sum_{\ell=1}^{L}\left(\int_{0}^{K_{\ell} / u_{\ell}^{\max }} u_{\ell}^{\max } \mathrm{d} F_{h_{\ell}(k)}\left(h_{\ell}\right)\right. \\
\left.+\int_{K_{\ell} / u_{\ell}^{\max }}^{\infty} \frac{K_{\ell}}{h_{\ell}} \mathrm{d} F_{h_{\ell}(k)}\left(h_{\ell}\right)\right) .
\end{array}
$$


It is easy to see that, since $f_{\ell}$ are monotonically increasing, for fixed maximum peak power levels $\left\{u_{1}^{\max }, \ldots, u_{L}^{\max }\right\}$, $\hat{\Phi}_{\ell}(k)$ and $\psi(\hat{\Phi}(k))$ are increasing functions of the gains $\left\{K_{1}, \ldots, K_{L}\right\}$. Thus, a necessary condition on the maximum peak power levels so that (19) can be satisfied is that

$$
\psi(\Upsilon(k)) \geq 1-\rho /\|A(k)\|^{2}, \quad \text { for some } \rho \in[0,1),
$$

where

$$
\Upsilon(k)=\left[\begin{array}{c}
\Upsilon_{1}(k) \\
\vdots \\
\Upsilon_{L}(k)
\end{array}\right], \Upsilon_{\ell}(k) \triangleq \int_{0}^{\infty} f_{\ell}\left(h_{\ell} u_{\ell}^{\max }\right) \mathrm{d} F_{h_{\ell}(k)}\left(h_{\ell}\right)
$$

On the other hand, the average sum power $\bar{U}(k)$ in (44) is also an increasing function of the gains and maximum peak power levels. Consequently, when designing constant gain saturated power control laws, there exists a trade-off between energy use and satisfaction of the sufficient condition for stability established in Theorem 3.

If $\left\{u_{1}^{\max }, u_{2}^{\max }, \ldots, u_{L}^{\max }\right\}$ are given, then the design problem consists in choosing the control gains $\left\{K_{1}, \ldots, K_{L}\right\}$. The optimization problem in (40) leads to:

$$
\min _{\left\{K_{1}, \ldots, K_{L}\right\}} \bar{U}(k), \quad \text { s.t. } \psi(\hat{\Phi}(k)) \geq 1-\rho /\|A(k)\|^{2},
$$

with $\hat{\Phi}_{\ell}(k)$ as in (43). This problem is, in general, nonconvex, since the objective function $\bar{U}(k)$ is actually a concave function of $\left\{K_{1}, \ldots, K_{L}\right\}$.

As an alternative to (46), one may also seek to optimize both $\left\{K_{1}, \ldots, K_{L}\right\}$ and $\left\{u_{1}^{\max }, \ldots, u_{L}^{\max }\right\}$, which is, again a non-convex optimization problem. In our numerical results documented in Section 7, these two optimization problems are addressed by using the NMinimize routine in Mathematica to find locally optimal solutions.

\subsection{Centralized Control}

With centralized control as in (8), the power levels $u_{\ell}(k)$ depend on the instantaneous channel power gains $h(k)$. The optimization problem in (40) can then be stated as, see (24),

$$
\begin{aligned}
& \min _{\left\{\kappa_{1}, \ldots, \kappa_{L}\right\}} \int_{\Omega} \sum_{\ell=1}^{L} \kappa_{\ell}(h) \mathrm{d} F_{h(k)}(h) \\
& \text { s.t. } \int_{\Omega} \sum_{\Gamma \in \mathbb{J}} \prod_{\ell=1}^{L} \Xi_{\ell}(h, \Gamma) \mathrm{d} F_{h(k)}(h) \geq 1-\frac{\rho}{\|A(k)\|^{2}}
\end{aligned}
$$

where $\Xi_{\ell}(h, \Gamma) \triangleq\left(1-\delta_{L}^{\ell} \Gamma\right)\left(1-\hat{\varphi}_{\ell}(h)\right)+\delta_{L}^{\ell} \Gamma \hat{\varphi}_{\ell}(h)$ and $\hat{\varphi}_{\ell}(h)$ is as in (33). The optimization problem (47) is not convex. To compute (sub-optimal) solutions, we use the generalized Karush-Kuhn-Tucker (KKT) conditions [24], which are necessary conditions for optimality. The Lagrangian is

$$
\begin{aligned}
\mathcal{L}\left(\kappa_{1}, \kappa_{2}, \ldots, \kappa_{L}, \nu\right) & =\sum_{\ell=1}^{L} \kappa_{\ell}(h) \\
& -\nu\left(\sum_{\Gamma \in \mathbb{J}} \prod_{\ell=1}^{L} \Xi_{\ell}(h, \Gamma)-\left(1-\frac{\rho}{\|A(k)\|^{2}}\right)\right) .
\end{aligned}
$$

Thus, the generalized KKT conditions become ${ }^{4}$

$$
\begin{gathered}
1-\nu f_{\ell}^{\prime}\left(h_{\ell} \kappa_{\ell}(h)\right) h_{\ell}\left(\sum_{\Gamma \in \mathbb{J}}\left(2 \delta_{L}^{\ell} \Gamma-1\right) \prod_{j \neq \ell} \Xi_{j}(h, \Gamma)\right)=0, \\
\int_{\Omega} \sum_{\Gamma \in \mathbb{J}} \prod_{\ell=1}^{L} \Xi_{j}(h, \Gamma) \mathrm{d} F_{h(k)}(h)=1-\frac{\rho}{\|A(k)\|^{2}}, \\
\nu \geq 0,
\end{gathered}
$$

where $\ell \in\{1, \ldots, L\}$. For given $\nu$ and $h$, we can solve the system of $L$ equations

$1-\nu f_{\ell}^{\prime}\left(h_{\ell} \kappa_{\ell}(\nu, h)\right) h_{\ell}\left(\sum_{\Gamma \in \mathbb{J}}\left(2 \delta_{L}^{\ell} \Gamma-1\right) \prod_{j \neq \ell} \Xi_{j}(h, \Gamma)\right)=0$

numerically for $\left\{\kappa_{1}(\nu, h), \ldots, \kappa_{L}(\nu, h)\right\}$. Amongst all possible values of $\nu$, we then find a value $\nu^{*}$ that satisfies the constraint with equality. A sub-optimal solution to the centralized control design problem (47) is then given by $\left\{\kappa_{1}\left(\nu^{*}, h\right), \ldots, \kappa_{L}\left(\nu^{*}, h\right)\right\}$.

Remark 18 As the optimization problem is not convex, the generalized KKT conditions (48) are necessary but not sufficient conditions for optimality. As an example consider the case of $L=1$ channel, $\mathbb{J}=\{1\}$, and BPSK transmission with $b=16$ bits per packet, see Example 1.The generalized KKT conditions give that $\kappa_{1}\left(\nu, h_{1}\right)$ should satisfy

$$
1-\nu f_{1}^{\prime}\left(h_{1} \kappa_{1}\left(\nu, h_{1}\right)\right) h_{1}=0
$$

Now suppose we have $\nu=10$, and $h_{1}=1$. Then we find that $\kappa_{1}\left(\nu, h_{1}\right)=9.713 \times 10^{-7}, \kappa_{1}\left(\nu, h_{1}\right)=0.7438$ and $\kappa_{1}\left(\nu, h_{1}\right)=4.9324$ all satisfy (49). Thus, (49) cannot be solved uniquely.

\section{Numerical Results}

To illustrate properties of the power allocation strategies discussed above, we next present some numerical results.

\footnotetext{
${ }^{4}$ We write $h_{\ell}$ for $\delta_{\ell}^{L} h$.
} 


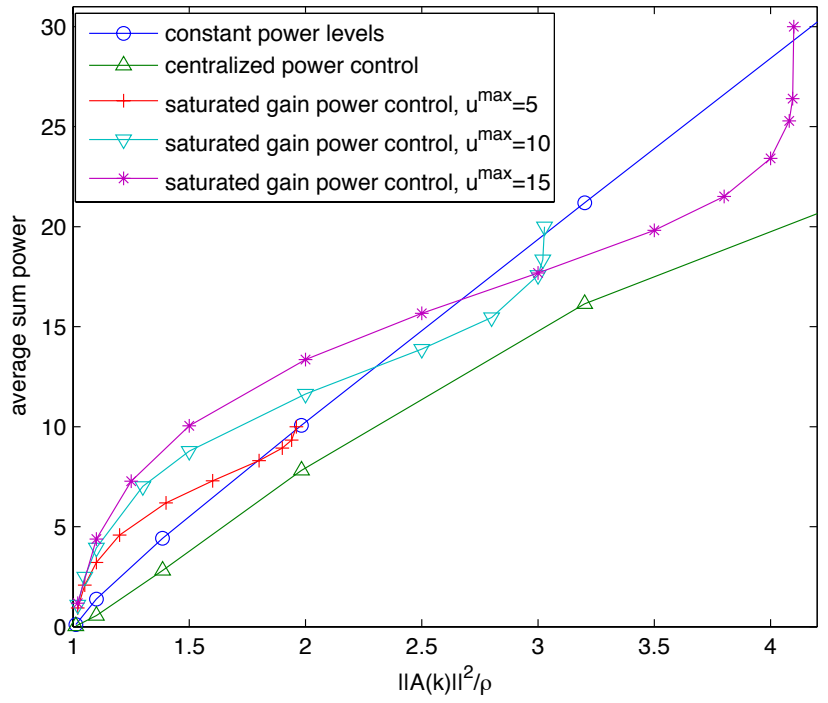

Fig. 2. Average sum power required to meet the stability condition (19) for the two-channel topology.

\subsection{Two-channel Topology}

Consider the estimation architecture described in Example 16, with $L=n=2$ Rayleigh fading channels with $\lambda_{1}=1$ and $\lambda_{2}=2$, and BPSK transmission with $b=4$ bits, see Example 1. Fig. 2 depicts the average sum power required as a function of $\|A(k)\|^{2} / \rho$ for the following control strategies: optimized constant power levels (see Section 6.1), saturated constant gain power control for given saturation values (see Section 6.2), and (sub)-optimal centralized control (see Section 6.3). Clearly, centralized power control outperforms the simpler decentralized schemes. Interestingly, for small values of $\|A(k)\|^{2} / \rho$, the saturated gain power controllers (with $u_{1}^{\max }=u_{2}^{\max }=u^{\max }$ ) perform worse than using constant power levels, designed as per (42); for larger values of $\|A(k)\|^{2} / \rho$, the saturated gain controllers perform better. One possible interpretation of this observation could be that the stability condition is easier to be met when $\|A(k)\|^{2} / \rho$ is small, whereas doing channel inversion in the saturated gain controller will result in increased power usage. However, as $\|A(k)\|^{2} / \rho$ approaches the maximum value where the stability condition can still be satisfied, see (45), the saturated gain power controllers again perform worse, since as $K_{\ell} \rightarrow \infty$, the policy (30) approaches a policy with constant powers $\left\{u_{1}^{\max }, \ldots, u_{L}^{\max }\right\}$, but which are not necessarily the optimal constant power levels.

\subsection{Estimation with Three Sensors}

We next consider the topology in Fig. 1 with BPSK transmission and Rayleigh fading channels with $\lambda_{1}=1, \lambda_{2}=2$ and $\lambda_{3}=1.5$, see Example 10. Fig. 3 compares the average sum power required under the three power control schemes examined in Section 6. It is easy to see that, if the power levels are allowed to depend upon the actual channel gains, then less sum power is needed to preserve the stabil-

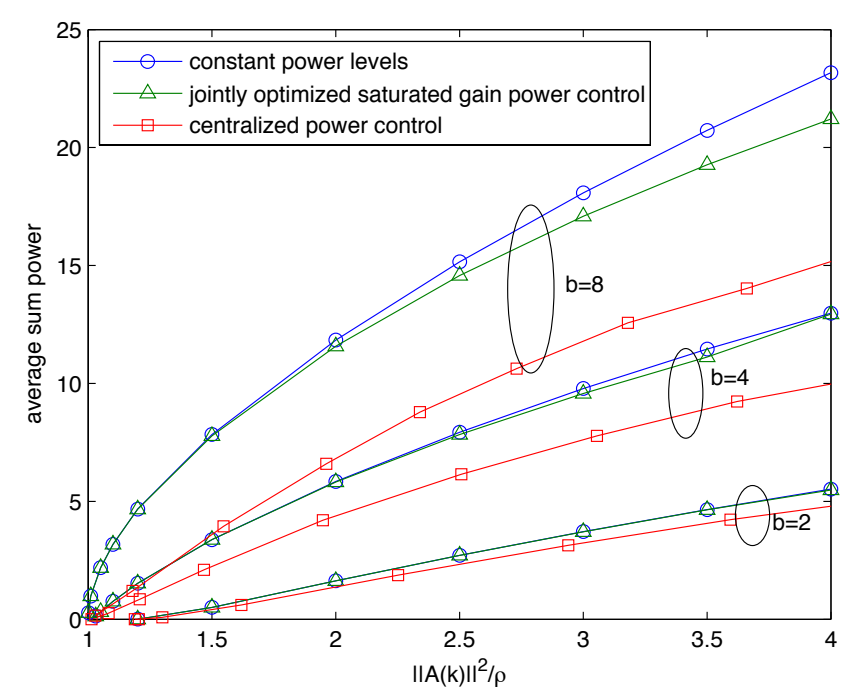

Fig. 3. Average sum power required to meet the stability condition (19) for the topology in Fig. 1.

ity condition given in Theorem 3 . The performance of the saturated gain power controller lies between the case with static power levels and the case with channel gain dependent power levels. Note that static power levels can be regarded as a special case of the saturated gain controller by setting $K_{\ell}=\infty$, which explains why the jointly optimized saturated gain power controller will perform better than the case with static power levels. Note that, as the number of bits per packet, $b$, and $\|A(k)\|^{2} / \rho$ increase, the performance gains of using time-varying power levels become more pronounced. For example, in the situation examined, if $\|A(k)\|^{2} / \rho=4$ and $b=8$ bits, then using optimized constant power levels requires more than $50 \%$ additional average sum power than using centralized (sub)-optimal power control.

\section{Conclusions}

We have studied stability of a Kalman filter receiving sensor data from a linear time-varying system over block fading wireless channels governed by power control. By the use of stochastic stability methods, we have established conditions on system parameters which ensure that the Kalman filter covariance matrix is exponentially bounded in norm. The stability condition obtained was then used as a constraint to obtain the minimum averaged sum power for different types of power control policies. Numerical studies indicate that channel gain dependent power allocation can significantly outperform the allocation of static powers which depend upon the channel statistics only. Future work could include further investigating optimal power control policies given different information available at the gateway. Also of interest would be the incorporation of source coding effects and allowing for correlated wireless channel models. Preliminary results on the latter have been documented in [33]. It is also of interest to consider more general wireless sensor network architectures, including those where the sensors have local processing capability as studied, e.g., in [12, 15, 27, 28, 37, 40, 42]. 


\section{A Proof of Theorem 3}

By Assumption 2, $\{\xi\}_{\mathbb{N}_{0}}$ defined in (15) is white. Thus, it follows from (6), (8) and (13) that $\{C\}_{\mathbb{N}_{0}}$ are independent, so that $\{P\}_{\mathbb{N}_{0}}$ in (14) is Markovian; see, e.g, [22, Chap. 2]. We next adopt a stochastic Lyapunov function approach with candidate function $V_{k} \triangleq \operatorname{tr} P(k) \geq 0, \forall k \in \mathbb{N}_{0}$, to show that there exist $\beta^{\prime} \in(0, \infty)$ and $\rho \in[0,1)$ such that

$$
\mathbf{E}\left\{V_{k+1} \mid P(k)\right\} \leq \rho V_{k}+\beta^{\prime}, \quad \forall k \in \mathbb{N}_{0} .
$$

For that purpose, we condition on $s(k)$ and write:

$$
\begin{gathered}
\mathbf{E}\left\{V_{k+1} \mid P(k)\right\}=\mathbf{E}\left\{\mathbf{E}\left\{V_{k+1} \mid P(k), s(k)\right\}\right\} \\
=\mathbf{E}\left\{V_{k+1} \mid P(k), s(k)=1\right\} \operatorname{Pr}\{s(k)=1\} \\
\quad+\mathbf{E}\left\{V_{k+1} \mid P(k), s(k)=0\right\} \operatorname{Pr}\{s(k)=0\} .
\end{gathered}
$$

For $s(k)=1, C(k)$ has full column-rank and a simple state prediction is $\bar{x}(k+1)=A(k)\left(C(k)^{T} C(k)\right)^{-1} C(k)^{T} y(k)$. Since $Q(k), R(k)$ and $A(k)$ are assumed bounded, the covariance of $x(k+1)-\bar{x}(k+1)$ can be uniformly bounded by $W I_{n}$, for some $W \in(0, \infty)$. Since the Kalman filter gives the minimum conditional estimation error covariance and the expectation and the trace operators commute, it holds that $\mathbf{E}\left\{V_{k+1} \mid P(k), s(k)=1\right\} \leq n W$. Thus,

$$
\mathbf{E}\left\{V_{k+1} \mid P(k), s(k)=1\right\} \operatorname{Pr}\{s(k)=1\} \leq n W .
$$

If $s(k)=0$, then $P(k+1)$ can be upper bounded by the covariance matrix of the estimation error resulting from the worst case, i.e., where $\gamma_{\ell}(k)=0$, for all $\ell \in\{1,2, \ldots, L\}$ :

$$
\begin{aligned}
& \left.\mathbf{E}\left\{V_{k+1} \mid P(k), s(k)=0\right\} \leq \mathbf{E}\left\{V_{k+1} \mid P(k), C(k)=0\right\}\right\} \\
& =\operatorname{tr}\left(A(k) P(k) A(k)^{T}+Q(k)\right) \leq\|A(k)\|^{2} V_{k}+q, \text { (A.4) }
\end{aligned}
$$

where we have used (14) and [1, Fact 8.12.29] and where $q \triangleq \max _{k \in \mathbb{N}_{0}} \operatorname{tr} Q(k)$. If we now substitute (A.3) and (A.4) into (A.2) and set $\beta^{\prime}=W n+q$, then we obtain $\mathbf{E}\left\{V_{k+1} \mid P(k)\right\} \leq \operatorname{Pr}\{s(k)=0\}\|A(k)\|^{2} V_{k}+\beta^{\prime}$ and (19) gives (A.1). It now follows from [25, Prop. 3.2], that $\mathbf{E}\left\{V_{k} \mid P(0)\right\} \leq \rho^{k} V_{0}+\beta^{\prime} /(1-\rho)$. Since $P(k) \succeq 0$, we have $V_{k}=\operatorname{tr} P(k) \geq\|P(k)\|$ for all $k \in N_{0}$.

\section{B Proof of Lemma 5}

By (21), the event $\{s(k)=1\}$ can be written as the sum of mutually exclusive events:

$$
\{s(k)=1\}=\sum_{\Gamma \in \mathbb{J}}\{\gamma(k)=\Gamma\} .
$$

Now, Assumption 4 and Eq. (6) give that, for all $\Gamma \in \mathbb{J}$,

$$
\begin{aligned}
& \operatorname{Pr}\{\gamma(k)=\Gamma \mid h(k)=h, u(k)=u\} \\
& =\prod_{\ell=1}^{L}\left(\left(1-\delta_{L}^{\ell} \Gamma\right)\left(1-f_{\ell}\left(h_{\ell} u_{\ell}\right)\right)+\delta_{L}^{\ell} \Gamma f_{\ell}\left(h_{\ell} u_{\ell}\right)\right),
\end{aligned}
$$

where $h_{\ell}=\delta_{L}^{\ell} h$ and $u_{\ell}=\delta_{L}^{\ell} u$. Expression (B.1) thus gives:

$$
\begin{aligned}
& \operatorname{Pr}\{s(k)=1 \mid h(k)=h, u(k)=u\} \\
& =\sum_{\Gamma \in \mathbb{J}} \prod_{\ell=1}^{L}\left(\left(1-\delta_{L}^{\ell} \Gamma\right)\left(1-f_{\ell}\left(h_{\ell} u_{\ell}\right)\right)+\delta_{L}^{\ell} \Gamma f_{\ell}\left(h_{\ell} u_{\ell}\right)\right) .
\end{aligned}
$$

The result follows from substitution of the control policy (8) - (11), Assumption 2 and the law of total probability.

\section{Proof of Lemma 9}

Since the control laws and channel estimates are decentralized, Equation (24) of Lemma 5 allows us to write:

$$
\begin{aligned}
\operatorname{Pr}\{s(k)=1\}= & \int_{\Omega \times \Lambda} \sum_{\Gamma \in \mathbb{J}} \prod_{\ell=1}^{L}\left(\left(1-\delta_{L}^{\ell} \Gamma\right)\left(1-\varphi_{\ell}\left(\xi_{\ell}\right)\right)\right. \\
& \left.+\delta_{L}^{\ell} \Gamma \varphi_{\ell}\left(\xi_{\ell}\right)\right) \mathrm{d} F_{\xi_{\ell}(k)}\left(\xi_{\ell}\right) \\
= & \sum_{\Gamma \in \mathbb{J}} \prod_{\ell=1}^{L} \int_{\Omega_{\ell} \times \Lambda_{\ell}}\left(\left(1-\delta_{L}^{\ell} \Gamma\right)\left(1-\varphi_{\ell}\left(\xi_{\ell}\right)\right)\right. \\
& \left.+\delta_{L}^{\ell} \Gamma \varphi_{\ell}\left(\xi_{\ell}\right)\right) \mathrm{d} F_{\xi_{\ell}(k)}\left(\xi_{\ell}\right) .
\end{aligned}
$$

Use of (27) and (29), thus gives

$$
\operatorname{Pr}\{s(k)=1\}=\sum_{\Gamma \in J} \prod_{\ell=1}^{L} \begin{cases}1-\Phi_{\ell}(k), & \text { if } \delta_{L}^{\ell} \Gamma=0 \\ \Phi_{\ell}(k), & \text { if } \delta_{L}^{\ell} \Gamma=1\end{cases}
$$

This proves the result.

\section{Proof of Theorem 12}

By Assumption 11, one can bound

$$
\begin{aligned}
& \left|f_{\ell}\left(\delta_{L}^{\ell} h \kappa_{\ell}(h)\right)-f_{\ell}\left(\delta_{L}^{\ell} h \kappa_{\ell}\left(\left(I_{L}+\operatorname{diag}(\epsilon)\right) h\right)\right)\right| \\
& \quad \leq \theta_{\ell}\left(\delta_{L}^{\ell} h\left|\kappa_{\ell}(h)-\kappa_{\ell}\left(\left(I_{L}+\operatorname{diag}(\epsilon)\right) h\right)\right|\right) \\
& \quad \leq \theta_{\ell}\left(\delta_{L}^{\ell} h \sigma_{\ell}\left(\left|h-\left(I_{L}+\operatorname{diag}(\epsilon)\right) h\right|\right)\right) \\
& \quad=\theta_{\ell}\left(\delta_{L}^{\ell} h \sigma_{\ell}(|\operatorname{diag}(\epsilon) h|)\right)
\end{aligned}
$$

which directly gives

$$
\begin{aligned}
& f_{\ell}\left(\delta_{L}^{\ell} h \kappa_{\ell}\left(\left(I_{L}+\operatorname{diag}(\epsilon)\right) h\right)\right) \\
& \quad \geq f_{\ell}\left(\delta_{L}^{\ell} h \kappa_{\ell}(h)\right)-\theta_{\ell}\left(\delta_{L}^{\ell} h \sigma_{\ell}(|\operatorname{diag}(\epsilon) h|)\right) .
\end{aligned}
$$

The bound (35) now follows from integrating (D.1) with respect to $\mathrm{d} F_{\xi(k)}$ and noting that $\int_{\Lambda} \mathrm{d} F_{\epsilon(k)}=1$. For decentralized control policies, (36) follows directly from (35) upon noting that $\operatorname{Pr}\{s(k)=1\}$ is minimized when $\operatorname{Pr}\left\{\gamma_{\ell}(k)=1\right\}$ are at their lowest values. 


\section{E Proof of Lemma 13}

For non-increasing decentralized control policies $\kappa_{\ell}$ and since $f_{\ell}$ are non-decreasing, $f_{\ell}\left(h_{\ell} \kappa_{\ell}\left(\left(1+\epsilon_{\ell}\right) h_{\ell}\right)\right) \geq$ $f_{\ell}\left(h_{\ell} \kappa_{\ell}\left(h_{\ell}\right)\right), \forall \epsilon_{\ell} \leq 0$, i.e., underestimating the channel gains does not reduce the success probabilities. Thus,

$$
\begin{aligned}
\Phi_{\ell}(k) \geq & \int_{\Omega_{\ell} \times\left(\Lambda_{\ell} \backslash \Lambda_{\ell}^{+}\right)} f_{\ell}\left(h_{\ell} \kappa_{\ell}\left(h_{\ell}\right)\right) \mathrm{d} F_{\xi_{\ell}(k)}\left(\xi_{\ell}\right) \\
& +\int_{\Omega_{\ell} \times \Lambda_{\ell}^{+}} f_{\ell}\left(h_{\ell} \kappa_{\ell}\left(\left(1+\epsilon_{\ell}\right) h_{\ell}\right) \mathrm{d} F_{\xi_{\ell}(k)}\left(\xi_{\ell}\right)\right. \\
= & \hat{\Phi}_{\ell}(k) \int_{\Lambda_{\ell} \backslash \Lambda_{\ell}^{+}} \mathrm{d} F_{\epsilon_{\ell}(k)} \\
& +\int_{\Omega_{\ell} \times \Lambda_{\ell}^{+}} f_{\ell}\left(h_{\ell} \kappa_{\ell}\left(\left(1+\epsilon_{\ell}\right) h_{\ell}\right) \mathrm{d} F_{\xi_{\ell}(k)}\left(\xi_{\ell}\right) .\right.
\end{aligned}
$$

On the other hand, (D.1) provides that

$$
\begin{aligned}
& \int_{\Omega_{\ell} \times \Lambda_{\ell}^{+}} f_{\ell}\left(h_{\ell} \kappa_{\ell}\left(\left(1+\epsilon_{\ell}\right) h_{\ell}\right) \mathrm{d} F_{\xi_{\ell}(k)}(\xi)\right. \\
& \geq \int_{\Omega_{\ell} \times \Lambda_{\ell}^{+}} f_{\ell}\left(h_{\ell} \kappa_{\ell}\left(h_{\ell}\right)\right) \mathrm{d} F_{\xi_{\ell}(k)}\left(\xi_{\ell}\right) \\
& \quad-\int_{\Omega_{\ell} \times \Lambda_{\ell}^{+}} \theta_{\ell}\left(h_{\ell} \sigma_{\ell}\left(\left|\epsilon_{\ell} h_{\ell}\right|\right)\right) \mathrm{d} F_{\xi_{\ell}(k)}\left(\xi_{\ell}\right) \\
& =\hat{\Phi}_{\ell}(k) \int_{\Lambda_{\ell}^{+}} \mathrm{d} F_{\epsilon_{\ell}(k)}-\int_{\Omega_{\ell} \times \Lambda_{\ell}^{+}} \theta_{\ell}\left(h_{\ell} \sigma_{\ell}\left(\epsilon_{\ell} h_{\ell}\right)\right) \mathrm{d} F_{\xi_{\ell}(k)}\left(\xi_{\ell}\right) .
\end{aligned}
$$

Substitution of (E.2) into (E.1) shows that (35) holds with $\Delta_{\ell}(k)=\Delta_{\ell}^{\prime}(k)$. This proves the result.

\section{F Proof of Corollary 14}

Expressions (38) and (36) give that

$\operatorname{Pr}\{s(k)=1\}-\psi(\hat{\Phi}(k)) \geq \psi(\hat{\Phi}(k)-\Delta(k))-\psi(\hat{\Phi}(k))$.

Now, $\psi$ in (29) is differentiable. Thus, we can use the meanvalue theorem to obtain:

$$
\operatorname{Pr}\{s(k)=1\}-\psi(\hat{\Phi}(k)) \geq-\nabla \psi(\tilde{\Phi}(k)) \Delta(k)^{T},
$$

where $\tilde{\Phi}(k)=\hat{\Phi}(k)-c \Delta(k)$, for some $c \in[0,1]$. Use of (38) and (37), therefore provides

$$
\operatorname{Pr}\{s(k)=1\} \geq 1-\frac{\tilde{\rho}}{\|A(k)\|^{2}}-D(k),
$$

Thus, the sufficient condition for exponential boundedness established in Theorem 3 is satisfied if $\exists \rho \in[0,1)$ such that

$$
1-\tilde{\rho} /\|A(k)\|^{2}-D(k) \geq 1-\rho /\|A(k)\|^{2},
$$

which is equivalent to (39).

\section{G Proof of Lemma 17}

Since the objective function is linear, (42) is convex if each of the functions $\hat{\Phi}_{\ell}(k)$ are log-concave functions of $u_{\ell}$. By properties of log-concave functions (see, e.g., [2, p.106]), a sufficient condition for this is that both the functions $\tilde{f}_{\ell}(h, u) \triangleq f_{\ell}(h u)$ and the derivatives of $F_{h_{\ell}(k)}(h)$ are logconcave functions in $(h, u)$.

For BPSK transmission with Rayleigh fading as presented in Example 1, the log-concavity of the derivative of $F_{h_{\ell}(k)}(h)$, namely $\lambda_{\ell} e^{-\lambda_{\ell} h}$ is obvious. Since log-concave functions are closed under multiplication, it suffices to show that $g(h, u) \triangleq(1 / \sqrt{2 \pi}) \int_{0}^{\sqrt{h u}} e^{-t^{2} / 2} \mathrm{~d} t$ is log-concave in $(h, u)$ for $h, u \in \mathbb{R}_{\geq 0}$. Now the second order condition for logconcavity says that a function is log-concave if and only if

$\nabla g \nabla g^{T}-g \nabla^{2} g=\left[\begin{array}{cc}\left(\frac{\partial g}{\partial h}\right)^{2}-g \frac{\partial^{2} g}{\partial h^{2}} & \frac{\partial g}{\partial h} \frac{\partial g}{\partial u}-g \frac{\partial^{2} g}{\partial h \partial u} \\ \frac{\partial g}{\partial u} \frac{\partial g}{\partial h}-g \frac{\partial^{2} g}{\partial u \partial h} & \left(\frac{\partial g}{\partial u}\right)^{2}-g \frac{\partial^{2} g}{\partial u^{2}}\end{array}\right] \geq 0$

After some computations, we can find that

$$
\begin{gathered}
\left(\frac{\partial g}{\partial h}\right)^{2}-g \frac{\partial^{2} g}{\partial h^{2}}=\frac{e^{-h u} u^{2}}{16 \pi(h u)^{3 / 2}} \\
\times\left(2 \sqrt{h u}+e^{h u / 2} \sqrt{2 \pi}(1+h u)(1+\operatorname{erf}(\sqrt{h u / 2}))\right) \geq 0,
\end{gathered}
$$

where $\operatorname{erf}(x) \triangleq(2 / \sqrt{\pi}) \int_{0}^{x} e^{-t^{2}} \mathrm{~d} t$ and

$$
\begin{gathered}
\left(\left(\frac{\partial g}{\partial h}\right)^{2}-g \frac{\partial^{2} g}{\partial h^{2}}\right)\left(\left(\frac{\partial g}{\partial u}\right)^{2}-g \frac{\partial^{2} g}{\partial u^{2}}\right) \\
-\left(\frac{\partial g}{\partial h} \frac{\partial g}{\partial u}-g \frac{\partial^{2} g}{\partial h \partial u}\right)^{2}=\frac{e^{-3 h u / 2}(1+\operatorname{erf}(\sqrt{h u / 2}))}{32 \pi^{2} h u} \\
\times\left(e^{h u / 2} \pi h u+\sqrt{2 \pi h u}+e^{h u / 2} \pi h u \operatorname{erf}(\sqrt{h u / 2})\right) \geq 0 .
\end{gathered}
$$

Thus, Sylvester's criterion gives that $\nabla g \nabla g^{T}-g \nabla^{2} g \geq 0$, for all $h, u \in \mathbb{R}_{\geq 0}$.

\section{References}

[1] D. S. Bernstein. Matrix Mathematics. Princeton University Press, Princeton, N.J., 2nd edition, 2009.

[2] S. Boyd and L. Vandenberghe. Convex Optimization. Cambridge University Press, 2004.

[3] R. Buche and H. J. Kushner. Control of mobile communications with time-varying channels in heavy traffic. IEEE Trans. Automat. Contr., 47(6):992-1003, June 2002.

[4] G. Caire, G. Taricco, and E. Biglieri. Optimum power control over fading channels. IEEE Trans. Inform. Theory, 45(5):1468-1489, July 1999. 
[5] A. Censi. Kalman filtering with intermittent observations: Convergence for semi-Markov chains and an intrinsic performance measure. IEEE Trans. Automat. Contr., 56(2):376-381, February 2011.

[6] C. D. Charalambous, S. M. Djouadi, and S. Z. Denic. Stochastic power control for wireless networks via SDEs: Probabilistic QoS measures. IEEE Trans. Automat. Contr., 51(12):4396-4401, December 2005

[7] S. Dey, A. S. Leong, and J. S. Evans. Kalman filtering with faded measurements. Automatica, 45(10):2223-2233, October 2009.

[8] T. Ekman. Prediction of Mobile Radio Channels: Modeling and Design. PhD thesis, Uppsala University, http://www.signal.uu.se/Publications/ptheses.html, October 2002.

[9] M. Epstein, L. Shi, A. Tiwari, and R. M. Murray. Probabilistic performance of state estimation across a lossy network. Automatica, 44(12):3046-3053, December 2008

[10] H. Gharavi and S. P. Kumar. Special section on sensor networks and applications. Proc. IEEE, 91(8):1151-1152, August 2003.

[11] F. Gunnarsson and F. Gustafsson. Control theory aspects of power control in UMTS. Contr. Eng. Pract., 11:1113-1125, 2003.

[12] V. Gupta, N. C. Martins, and J. S. Baras. Optimal output feedback control using two remote sensors over erasure channels. IEEE Trans. Automat. Contr., 54(7):1463-1476, July 2009.

[13] S. V. Hanly and D.-N. Tse. Power control and capacity of spread spectrum wireless networks. Automatica, 35:1987-2012, 1999.

[14] P. Havinga, J. C. Hou, and F. Zhao. Wireless sensor networks [guest ed.]. IEEE Wireless Commun., 11(12):4-5, December 2004.

[15] J. P. Hespanha, P. Naghshtabrizi, and Y. Xu. A survey of recent results in networked control systems. Proc. IEEE, 1(95):138-162, January 2007.

[16] M. Huang, P. E. Caines, and R. P. Malhamé. Uplink power adjustment in wireless communication systems: A stochastic control analysis. IEEE Trans. Automat. Contr., 49(10):1693-1708, October 2004.

[17] M. Huang and S. Dey. Stability of Kalman filtering with Markovian packet losses. Automatica, 43(4):598-607, April 2007.

[18] M. Ilyas, I. Mahgoub, and L. Kelly. Handbook of Sensor Networks: Compact Wireless and Wired Sensing Systems. CRC-Press, Inc, Boca Raton, FL, USA, 2004.

[19] Z. Jin, V. Gupta, and R. Murray. State estimation over packet dropping networks using multiple description coding. Automatica, 42(9):1441-1452, September 2006.

[20] S. Kar, B. Sinopoli, and J. M. F. Moura. Kalman filtering with intermittent observations: Weak convergence to a stationary distribution. IEEE Trans. Automat. Contr., 2011. to be published.

[21] S. Kluge, K. Reif, and M. Brokate. Stochastic stability of the extended Kalman filter with intermittent observations. IEEE Trans. Automat. Contr., 55(2):514-518, February 2010.

[22] P. R. Kumar and P. Varaiya. Stochastic Systems: Estimation, Identification and Adaptive Control. Prentice Hall, Englewood Cliffs, N. J., 1986.

[23] X. Liu and A. Goldsmith. Kalman filtering with partial observation losses. In Proc. IEEE Conf. Decis. Contr., pages 4180-4186, Paradise Island, Bahamas, 2004.

[24] D. G. Luenberger. Optimization by Vector Space Methods. WileyInterscience, New York, N.Y., 1969.

[25] S. P. Meyn. Ergodic theorems for discrete time stochastic systems using a stochastic Lyapunov function. SIAM Journal on Control and Optimization, 27(6):1409-1439, November 1989.

[26] Y. Mostofi and R. M. Murray. To drop or not to drop: Design principles for Kalman filtering over wireless fading channels. IEEE Trans. Automat. Contr., 54(2):376-381, February 2009.
[27] E. J. Msechu, S. I. Roumeliotis, A. Ribeiro, and G. B. Giannakis. Decentralized quantized Kalman filtering with scalable communication cost. IEEE Trans. Signal Processing, 56(8):37273741, August 2008.

[28] J. Østergaard, D. E. Quevedo, and A. Ahlén. Predictive power control for dynamic state estimation over wireless sensor networks with relays. In Proc. 2010 Eur. Sig. Proc. Conf., Denmark, 2010.

[29] N. A. Pantazis and D. D. Vergados. A survey on power control issues in wireless sensor networks. IEEE Commun. Surv. Tutorials, 9(4):86-107, 2007.

[30] P. G. Park, C. Fischione, and K. H. Johansson. Experimental evaluation of power control algorithms for wireless sensor networks. In Proc. IFAC World Congr., Seoul, Korea, 2008.

[31] K. Plarre and F. Bullo. On Kalman filtering for detectable systems with intermittent observations. IEEE Trans. Automat. Contr., 54(2):386-390, February 2009.

[32] J. G. Proakis. Digital Communications. McGraw-Hill, New York, N.Y., 3rd edition, 1995.

[33] D. E. Quevedo, A. Ahlén, and K. H. Johansson. Stability of state estimation over sensor networks with Markovian fading channels. In Proc. IFAC World Congr., 2011.

[34] D. E. Quevedo, A. Ahlén, A. S. Leong, and S. Dey. On Kalman filtering with fading wireless channels governed by power control. In Proc. IFAC World Congr, pages 6586-6591, 2011.

[35] D. E. Quevedo, A. Ahlén, and J. Østergaard. Energy efficient state estimation with wireless sensors through the use of predictive power control and coding. IEEE Trans. Signal Processing, 58(9):48114823, September 2010.

[36] E. Rohr, D. Marelli, and M. Fu. Statistical properties of the error covariance in a Kalman filter with random measurement losses. In Proc. IEEE Conf. Decis. Contr., Atlanta, GA USA, 2010.

[37] L. Schenato. Optimal estimation in networked control systems subject to random delay and packet drop. IEEE Trans. Automat. Contr., 53(5):1311-1317, June 2008.

[38] L. Schenato, B. Sinopoli, M. Franceschetti, K. Poolla, and S. S. Sastry. Foundations of control and estimation over lossy networks. Proc. IEEE, 95(1):163-187, January 2007.

[39] X. Shen, Q. Zhang, and R. Caiming Qiu. Wireless sensor networking [guest ed.]. IEEE Wireless Commun., 14(6):4-5, December 2007.

[40] L. Shi, M. Epstein, and R. M. Murray. Kalman filtering over a packet-dropping network: A probabilistic perspective. IEEE Trans. Automat. Contr., 55(3):594-604, March 2010.

[41] B. Sinopoli, L. Schenato, M. Franceschetti, K. Poolla, M. I. Jordan, and S. S. Sastry. Kalman filtering with intermittent observations. IEEE Trans. Automat. Contr., 49(9):1453-1464, September 2004.

[42] A. Speranzon, C. Fischione, K. H. Johansson, and A. SangiovanniVincentelli. A distributed minimum variance estimator for sensor networks. IEEE J. Select. Areas Commun., 26(4):609-621, May 2008.

[43] Y. Xia, J. Shang, J. Chen, and G.-P. Liu. Networked data fusion with packet losses and variable delays. IEEE Trans. Syst., Man, Cybern. $B, 39(5): 1107-1120$, October 2009 .

[44] J.-J. Xiao, S. Cui, Z.-Q. Luo, and A. J. Goldsmith. Power scheduling of universal decentralized estimation in sensor networks. IEEE Trans. Signal Processing, 54(2):413-422, February 2006.

[45] L. Xie and L. Xie. Stability of a random Riccati equation with Markovian binary switching. IEEE Trans. Automat. Contr., 53(7):1759-1764, August 2008.

[46] X. Zhang, H. V. Poor, and M. Chiang. Optimal power allocation for distributed detection over MIMO channels in wireless sensor networks. IEEE Trans. Signal Processing, 56(9):4124-4140, September 2008. 